\title{
BMJ Open Economic impact of early intervention in psychosis services: results from a longitudinal retrospective controlled study in England
}

\author{
Apostolos Tsiachristas, ${ }^{1}$ Tony Thomas, ${ }^{2}$ Jose Leal, ${ }^{1}$ Belinda R Lennox ${ }^{3}$
}

To cite: Tsiachristas A, Thomas T, Leal J, et al. Economic impact of early intervention in psychosis services: results from a longitudinal retrospective controlled study in England. BMJ Open 2016;6:e012611. doi:10.1136/bmjopen-2016012611

- Prepublication history and additional material is available. To view please visit the journal (http://dx.doi.org/ 10.1136/bmjopen-2016012611).

Received 12 May 2016 Revised 24 August 2016 Accepted 6 September 2016

CrossMark

\footnotetext{
${ }^{1}$ Health Economics Research Centre, Nuffield Department of Population Health, University of Oxford, Oxford, UK

2Janssen Healthcare Innovation, Johnson \& Johnson, High Wycombe, UK ${ }^{3}$ Department of Psychiatry, University of Oxford, Warneford Hospital, Oxford, UK

\section{Correspondence to} Dr Belinda R Lennox; Belinda.lennox@psych.ox.ac. uk
}

\section{ABSTRACT}

Objectives: To demonstrate the costs, outcomes and economic impact of early intervention in psychosis (EIP) services.

Design: Longitudinal retrospective observational study of service usage and outcome data from mental health and acute care services for all those with a diagnosis of psychosis in contact with mental health services over a 3-year period (April 2010-March 2013).

Setting: Thames Valley and South Midlands region in England (region covered by Oxford Academic Health Science Network).

Participants: 3674 people with psychosis, aged 16-35 years.

Interventions: EIP team or other community mental health teams.

Main outcome measures: Change in housing status, change in employment status and improvement on each of the four domains of the Health of the Nation Outcome Scale (HONOS) questionnaire. Costs of mental and acute health inpatient, outpatient and community service use were also included in the study. Results: Patients in EIP services were $116 \%$ more likely ( $95 \% \mathrm{Cl} 1.263$ to 3.708 ) to gain employment, $52 \%$ more likely to become accommodated in a mainstream house (95\% $\mathrm{Cl} 0.988$ to 2.326 ) and $17 \%$ more likely to have an improvement in the emotional well-being domain of the HONOS questionnaire ( $95 \% \mathrm{Cl} 1.067$ to 1.285 ), as compared to those in non-EIP services. Annual National Health Service (NHS) costs were also significantly lower for patients using EIP services compared to non-users. The mean annual NHS cost savings associated with EIP were $£ 4031$ ( $95 \% \mathrm{Cl} £ 1281$ to £6780). These mostly came from lower mental health inpatient costs (£4075, $95 \% \mathrm{Cl} £ 1164$ to £6986), lower acute hospital outpatient costs (£59, 95\% Cl £9 to £109), lower accident and emergency costs ( $\_31,95 \% \mathrm{Cl} £ 12$ to $£ 51$ ), and higher mental health community costs ( $£ 648,95 \% \mathrm{Cl} £ 122$ to $£ 1175$ ). If all people with a first-episode psychosis across England were to be treated by EIP services, the savings in societal costs would be an estimated £63.3 million per year, of which £33.5 million would be in NHS costs.

Conclusions: Treatment within an EIP service is associated with better health and social outcomes, and reduced costs.

\section{Strengths and limitations of this study}

- The study used large data set over a 3-year period after linkage of data across care settings.

- Advanced statistical techniques used to analyse panel data and to control for confounding.

- Diagnosis of first-episode psychosis was not directly recorded in the electronic patient records.

- The direct comparability of the early intervention in psychosis (EIP) and non-EIP groups cannot be established conclusively, as would have been the case in a trial setting.

- The data lacked of quality of life measurements and the costs of setting up and running EIP services as standalone teams.

\section{INTRODUCTION}

Psychosis is a common and severe mental illness, with a large health and economic impact on societies worldwide. Early intervention in psychosis (EIP) services are community-based multidisciplinary teams that seek to reduce duration of untreated psychosis and improve outcomes. ${ }^{1}{ }^{2}$ They proactively engage young people and families in assessment and treatment, have low caseloads per care coordinator, enabling a crisis response to avoid admissions, and have strong links with employment and education settings to promote early recovery. EIP services are recommended by National Institute for Health and Care Excellence (NICE) for adults with psychosis ${ }^{3}$ and are the basis of the first 'Access and Waiting Time' standard for mental health to be introduced in England in April 2016. ${ }^{4}$ There is limited evidence on the cost-effectiveness of these services. The highest quality data, from randomised controlled trials, comes from two studies, in 144 participants in England ${ }^{5}$ and 547 participants in Denmark. ${ }^{6}$ They both demonstrate the potential cost savings of EIP services. However, it is not known 
whether these savings can also be demonstrated in 'realworld' services as currently implemented into routine clinical practice. EIP has been implemented in a variable manner in England. This variability, combined with the availability of large linked data sets, allows us to explore the impact of EIP services outside a clinical trial setting, with comparable groups of young people either receiving an EIP service or not, depending on their home address. Hence, we aim to estimate the economic impact of EIP services, as implemented in England, on individuals with a diagnosis of psychosis based on health and social outcomes and costs.

\section{METHODS}

\section{Study design and setting}

A longitudinal retrospective observational study was conducted to compare the costs and effects of EIP services with other community mental health teams (CMHTs) over a 3-year period (April 2010-March 2013) in the Oxford Academic Health Science Network (AHSN) region. The Oxford AHSN brings together universities, industry and the National Health Service (NHS) to improve health and prosperity in this region through rapid clinical innovation adoption and covers a population of 3.3 million living in Berkshire, Buckinghamshire, Milton Keynes, Oxfordshire and Bedfordshire. ${ }^{7}$ Care to this population is provided by 4 Mental Health Trusts and 7 Acute Hospital Trusts, and funded by 12 clinical commissioning groups (CCGs). There was variable provision of EIP services across the geography, with EIP services funded by only 6 of the 12 CCGs over this time period. This enables the natural comparison of outcomes associated with EIP and CMHTs in comparable patient groups. The EIP services that are in place are standalone community multidisciplinary teams, containing psychiatrists, psychologists and care coordinators of a variety of professional backgrounds, mostly community psychiatric nurses. They operate according to the Early Intervention in Psychosis (IRIS) guidelines for EIP services, ${ }^{8}$ namely low client-to-care coordinator ratio (1:15), enabling assertive community treatment, expecting to see each clients on a weekly basis. Psychological therapies and family therapy are offered to clients routinely and are delivered by staff within the team. Clients are treated for a period of 3 years. This includes relapse prevention work, undertaken after the initial acute illness, to enable discharge from mental health services, to General Practice (GP) follow-up, after this 3-year period. In contrast, care within CMHTs is delivered with a higher caseload per care coordinator (at least 1:30). Psychological therapies and family therapy are offered after referral to a separate psychological treatment service and involve a waiting list of several months. Clients are treated within CMHTs for the duration of their acute illness and then discharged once stable. The length of treatment by a CMHT for those with first-episode psychosis is 6-9 months.

\section{Patient sample}

We used routinely collected healthcare delivery data licenced from the Health and Social Care Information
Centre (HSCIC) by Janssen Healthcare Innovation (2013). Data included non-sensitive, pseudonymised linked information from Hospital Episode Statistics (HES-Inpatient, Outpatient and Accident and Emergency) and from Mental Health Minimum Data Set (MHMDS), across 3 NHS years (April 2010-March 2013; 2010-2011, 2011-2012 and 2012-2013). Ethics approval was not needed because data could not be linked to patients or providers. Patients belonging to the Oxford AHSN area were selected using their most recent reported residence locations-all those with the lower super output area of residence within one of Oxford AHSN's CCGs.

Briefly, we identified patients with psychosis using the MHMDS and linked the respective acute care data from HES. The approach to identify psychosis patients in the MHMDS database was developed with clinical experts and service leaders, and validated by analyses performed in 3 (of 15) AHSNs in England. Patients were identified with psychosis if they (1) had a primary or secondary diagnosis of psychosis (ICD-10 codes: F20 schizophrenia, F22 persistent delusional disorders or F25 schizoaffective disorders), (2) were classified into one of the Mental Healthcare Clusters related with psychosis (clusters 1017, using the Mental Health Clustering Tool) and (3) reported problems associated with hallucination and delusions (question 6 from Health of the Nation Outcome Scale (HONOS) in the HONOS Working Age Adults Questionnaire).

A total of 15709 patients with psychosis were identified compared to 17993 reported in quality and outcomes framework (QOF) data. ${ }^{9}$ We further restricted the sample to patients in the age group of 16-35 years with an observed HONOS score at 2010/2011, which resulted in 3674 patients. We identified users of the EIP services in the MHMDS using the mental healthcare team with assigned responsibility for the care of the patient. Hence, the final sample consisted of 831 patients who were treated under EIP service and 2843 patients who were under standard community care (ie, did not have any interaction with the EIP service in the 3-year period considered for analysis). A detailed description of the sample selection criteria and sample identification is presented in online supplementary appendix 1 .

\section{Healthcare costs, health and social outcomes}

In the base case analysis, we estimated the annual healthcare costs of EIP and non-EIP users. Hence, we valued the annual utilisation of the following healthcare services: inpatient length of stay (LOS) in mental health hospital wards, acute hospital emergency (A\&E) visits, planned (elective) and emergency (non-elective) LOS in acute care hospital wards, outpatient visits in mental healthcare and acute care, and community mental healthcare contacts. All unit costs concerned 2013/2014 prices and are presented in online supplementary appendix 2. 
Outcomes were measured in terms of becoming employed (ie, proportion of those unemployed at start of follow-up who became employed during follow-up), resuming studying (ie, proportion of those not in education at start that were in education during follow-up), moving to mainstream housing (not in mainstream housing at start and gaining housing during follow-up), stopping sleeping rough (homeless at start and not homeless at follow-up) and improvement on each of the four domains (ie, personal, emotional and social wellbeing and severe disturbance) of the HONOS questionnaire (the HONOS scores decreased during the follow-up). The HONOS scores on each domain were calculated as the sum of the scores of each related questions. ${ }^{10}$ The outcome variables were binary in nature (ie, Yes or No) and were calculated as the (earliest recorded to latest recorded) change in accommodation, employment status and HONOS scores between 2010/2011 and 2012/2013.

\section{Propensity score matching}

As a consequence of the observational study design, the patients using EIP and non-EIP services differed in severity and socio-demographic characteristics. Therefore, we used propensity score matching (PSM) to reduce confounding caused by these differences. The scores on the four dimensions of the HONOS score were used as indicators of well-being and disease severity, while age, gender (male/female) and white-British ethnicity (Yes/No) were used to reduce differences in sociodemographic characteristics that might independently affect outcome. Inverse probability weighting was chosen as the preferred PSM technique because of its suitability for heavily unbalanced and relatively small samples. ${ }^{11}$ Stabilised inverse propensity scores were used as weights to preserve the sample size of the original data and to produce appropriate estimations of variance. ${ }^{12}$

\section{Statistical analysis}

The mean annual costs per patient and per healthcare service in each of the three follow-up years and its difference between users of EIP and non-EIP services were estimated using generalised estimation equation (GEE) models. The GEE model assumes that data are missing at random and uses the available data to adjust the estimates from bias resulting from missing data that are related to observed variables (eg, more severely unwell patients have more missing data). We used a gamma distribution and $\log$ link for the cost estimation. The main covariates explored in the model were categorical: EIP services (reference category: Not-EIP service), financial years of 2011/2012 and 2012/2013 (reference category: 2010/2011) and interaction terms of the EIP and financial year. The mean predicted costs from each model were weighted by the stabilised inverse propensity scores.

The outcomes were analysed cross-sectionally as they were expressed in changes in accommodation, employment status and HONOS scores between the earliest recorded and latest recorded values within the 3-year follow-up period. Probability ratios were estimated by using generalised linear models (GLMs) with binomial distribution and log link and were weighted by the stabilised inverse propensity scores. Only the EIP binary covariate was included in the GLMs' main structure.

Considering the large number of missing observations in the most recent measurement of employment, accommodation, and HONOS variables, we performed multiple imputations. For each missing observation in any of the binary outcome variables, 100 imputed observations were generated based on the observation on the four

Table 1 Descriptive baseline characteristics of psychosis cohort before and after PSM ( $n=3674)$

\begin{tabular}{|c|c|c|c|c|}
\hline & \multicolumn{2}{|c|}{ Observed } & \multicolumn{2}{|c|}{ After PSM } \\
\hline & EIP & No-EIP & EIP & No-EIP \\
\hline $\mathrm{n}$ & 831 & 2843 & 831 & 2843 \\
\hline Age, mean (SD) & $24.72(4.62)$ & $27.83(4.99)$ & $27.03(4.95)$ & $27.11(5.12)$ \\
\hline \multicolumn{5}{|l|}{ HONOS score baseline } \\
\hline Personal well-being (min 0-max 15), mean (SD) & $3.47(2.83)$ & $3.49(2.94)$ & $3.40(2.80)$ & $3.47(2.94)$ \\
\hline Emotional well-being (min 0-max 12), mean (SD) & $3.68(2.29)$ & $4.34(2.74)$ & $4.00(2.33)$ & $4.18(2.73)$ \\
\hline Social well-being (min 0-max 16), mean (SD) & $4.06(2.94)$ & $4.19(3.18)$ & $4.04(2.91)$ & $4.15(3.17)$ \\
\hline Severe disturbance (min 0-max 8), mean (SD) & $2.29(1.78)$ & $2.33(1.93)$ & $2.29(1.77)$ & $2.32(1.93)$ \\
\hline Gender-male (\%) & 65.46 & 55.22 & 56.66 & 57.49 \\
\hline Ethnicity一white British (\%) & 56.68 & 64.58 & 60.65 & 62.48 \\
\hline \multicolumn{5}{|l|}{ Employment status } \\
\hline Employed (\%) & 12.24 & 11.95 & 12.75 & 11.62 \\
\hline Students in full/part-time education (\%) & 9.96 & 6.08 & 7.16 & 6.67 \\
\hline Missing (\%) & 48.62 & 50.98 & 50.15 & 50.68 \\
\hline \multicolumn{5}{|l|}{ Accommodation } \\
\hline Homeless (\%) & 4.44 & 4.01 & 4.77 & 4.15 \\
\hline Mainstream housing (\%) & 45.62 & 40.86 & 43.39 & 41.06 \\
\hline Missing (\%) & 42.26 & 46.80 & 44.58 & 46.31 \\
\hline
\end{tabular}


HONOS domains and age in $2010 / 2011$ as well as the binary variables male (Yes/No) and white-British ethnicity (Yes/No) by using a logistic regression. The imputations were generated separately for people in the EIP and non-EIP to avoid introducing biases due to different missing patterns in the two groups. The same GLMs as in the complete case analysis were then used to estimate the outcomes based on the imputed data set.

Parameter uncertainty in the estimated coefficients of the models was captured by bootstrapping 5000 times (with the replacement) the patient sample and re-estimating the regression models. Parameter uncertainty was then propagated using probabilistic sensitivity analysis. This consisted of using the derived sets of regression coefficients to estimate the distribution of predicted costs/outcomes. The CIs of the predicted mean costs/outcomes and mean cost/outcomes differences were obtained by the 2.5 th and 97.5 th centiles. All statistical analyses were performed in STATA V.13.

\section{Scenario analysis}

We explored the economic impact of providing EIP services to 16-65 years old people with a first episode of psychosis in England. This is the target population for EIP services in England as of April 2016 and amounts to a predicted 8321 new cases per year. ${ }^{13}$ The economywide perspective used here included healthcare costs, productivity gains and housing benefits (transfer payments). Probabilistic sensitivity analysis was used to derive $95 \%$ CI around the estimates. We estimated the annual gains and losses per cost category from providing EIP to this population. The change in employment status was translated into annual productivity gains by calculating the difference in the number of patients in employment between EIP and non-EIP services and multiplying it by the English mean income in the $<20$ -64 years age group weighted by the proportion of men and women in the sample. Based on the literature, we assumed that patients were employed on average for 0.65 full-time equivalent (FTE). ${ }^{14}$ The number of employed service users if no EIP services were provided was based on the proportion of those in the non-EIP service who were employed (before PSM). This number was then multiplied by the probability ratio of becoming employed in EIP service to calculate the total number of employed EIP service users. Similarly, annual savings in terms of reduced governmental expenditure for housing benefits (transfer payments) were calculated in the case where patients got a mainstream house.

\section{Sensitivity analyses}

Several sensitivity analyses were performed to investigate the sensitivity of the results to the underlying data and assumptions. First, we report our results based on the complete cases (ie, without imputing missing observations). Second, we estimated the effect size of EIP on the four HONOS scores on the continuous scale (ie, as the differences between the earliest and latest HONOS

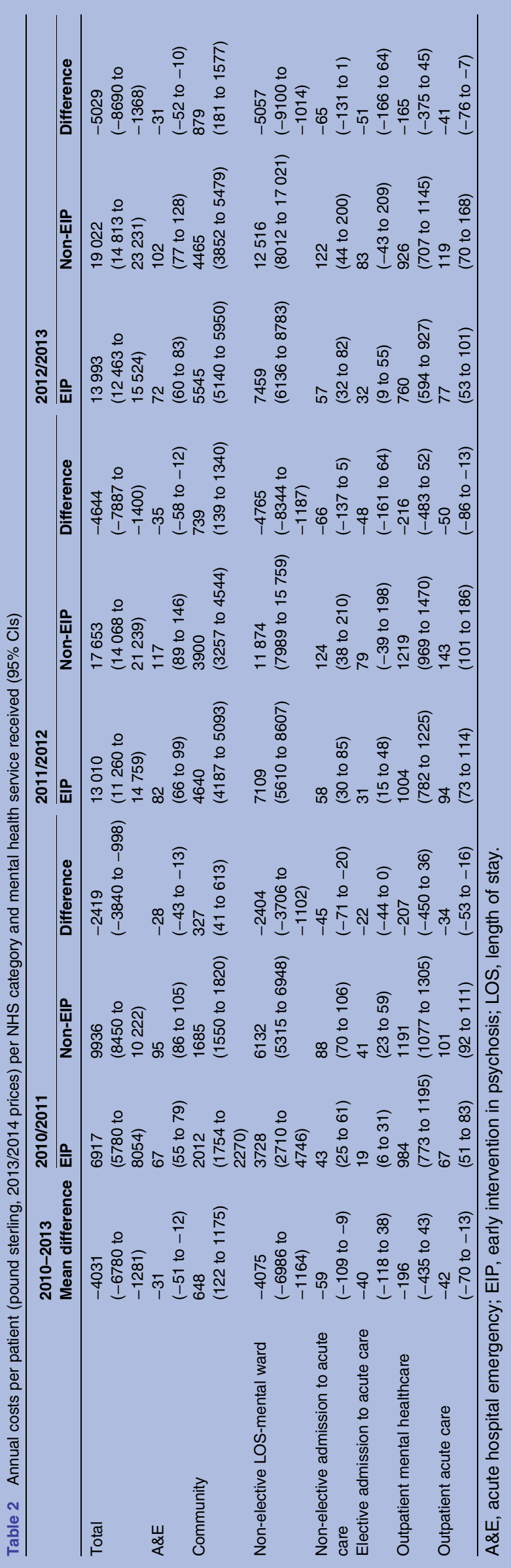


Figure 1 Mental health costs per patient per year and healthcare service.

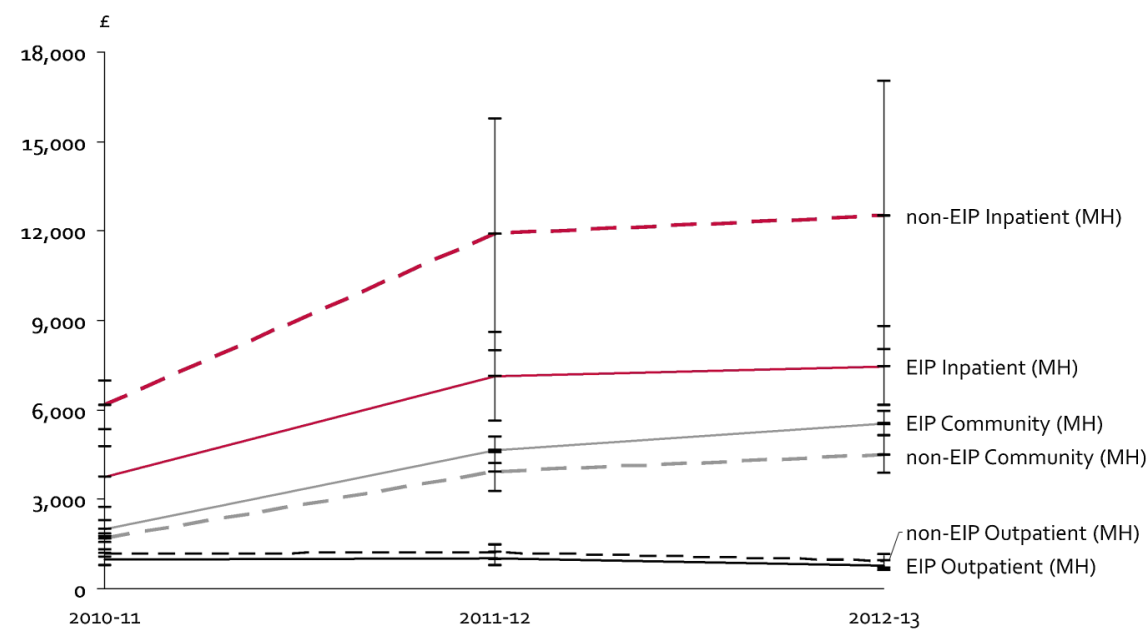

scores per patient) to investigate the impact of defining the HONOS outcome variables as binary. Third, we explored the sensitivity of the scenario results to the assumed employed FTE by varying it between 0.5 (lower assumed level) and 0.8 FTE (upper assumed level). Fourth, we performed the scenario analysis using patients aged 16-35 years as target population.

\section{RESULTS}

\section{Sample characteristics}

The descriptive statistics of the sample in 2010/2011 are presented in table 1, including the patient characteristics after PSM. The differences in patient characteristics between EIP and non-EIP samples after PSM were substantially reduced (eg, scores on three out of the four HONOS domains, being employed, and living in mainstream house) and in some cases were almost eliminated (eg, mean age, being white British). After applying PSM, patients in the EIP service were on average 27 years old and scored on average 3.40 on the personal well-being domain (out of 15), 4.00 on the emotional well-being domain (out of 12), 4.04 on the social well-being (out of 16) and 2.29 on the severe disturbance domain of HONOS (out of 8). Furthermore, $57 \%$ of the patients were men, $61 \%$ were white British, $13 \%$ were employed at the start of follow-up and $43 \%$ were living in a mainstream house at the start of follow-up.
Results from the main cost and outcome analysis

The annual costs per patient in EIP and non-EIP services for each year are reported in table 2 and figure 1 . The mean saving in NHS costs for each patient in EIP per year was $£ 4031$ (95\% CI £1281 to £6780). This was mainly driven by savings in non-elective LOS in mental healthcare (£4075). The mean savings in NHS costs from EIP service increased from £2419 in 2010/2011 to $£ 5029$ in 2012/2013. The differences in costs of elective healthcare between EIP and non-EIP services were not statistically significant.

Over the 3-year period and compared to patients in other CMHTs, patients in EIP services had almost $116 \%$ higher probability (95\% CI 1.263 to 3.708 ) of gaining employment, $52 \%$ higher probability of moving to mainstream housing (95\% CI 0.988 to 2.326 ) and $17 \%$ higher probability of having an improvement in emotional well-being domain of HONOS (95\% CI 1.067 to 1.285) (table 3).

\section{Results from the main scenario analysis}

The scenario analyses are presented in table 4. If EIP were to be provided to all $16-65$ years old people with a first-episode psychosis across England (ie, 8321 new cases per year), there would be 1161 (95\% CI -11 to 2330) more people in paid employment every year, with admissions to acute care and costs of outpatient mental

Table 3 Results of the outcome analysis for patients with psychosis $(n=3674)$

\begin{tabular}{lccccc}
\hline Outcome & Probability ratio & SE & p Value & Lower 95\% Cl & Upper 95\% Cl \\
\hline Becoming employed & 2.164 & 0.592 & 0.005 & 1.263 & 3.708 \\
Resuming studying & 1.828 & 0.775 & 0.156 & 0.793 & 4.212 \\
Moving to mainstream housing & 1.516 & 0.316 & 0.056 & 0.988 & 2.326 \\
No longer homeless & 1.618 & 0.481 & 0.107 & 0.901 & 2.903 \\
HONOS personal well-being improvement & 1.002 & 0.054 & 0.970 & 0.901 & 1.114 \\
HONOS emotional well-being improvement & 1.171 & 0.055 & 0.001 & 1.067 & 1.285 \\
HONOS social well-being improvement & 1.066 & 0.055 & 0.212 & 0.964 & 1.179 \\
HONOS severe disturbance improvement & 1.035 & 0.056 & 0.518 & 0.932 & 1.150 \\
HONOS, Health of the Nation Outcome Scale. & & & & &
\end{tabular}


resulting £21.7 million (95\% CI -0.2 to 43.6 ) productivity gains for the English economy. EIP services would also lead to 1734 (95\% CI -311 to 3798 ) more young people per year moving into mainstream housing, saving an estimated $£ 8$ million (95\% CI -1.4 to 17.6$)$ in housing benefits. The total savings/gains outside NHS (ie, including savings in housing benefits and productivity gains) would therefore be $£ 29.8$ (95\% CI 6.5 to 53.4) million per year. The total saving in NHS costs for providing EIP across England would be $\sim £ 33.5$ (95\% CI 10.4 to 55.1) million per year ( $£ 4031$ reduction in total costs times 8321 patients). Hence, the total saving/gains to overall English economy due to widespread implementation of EIP would be $£ 63.3$ (95\% CI 30.4 to 95.8) million per year.

\section{Results from the sensitivity analyses}

The results from the complete case analysis of the outcomes are presented in the upper panel of table 5 . These results were similar to the results from the main outcome analysis (see table 3), except for a lower probability ratio of getting employed if receiving EIP (from 2.164 in the main analysis to 1.989) and a higher $\mathrm{p}$ value of the outcome moving to mainstream housing (from 0.056 in the main analysis to 0.059 ). The results from the second sensitivity analysis are presented in the lower panel of table 5 . As these results show, patients in EIP had on average 0.545 (95\% CI 0.294 to 0.795$)$ and 0.246 (95\% CI 0.048 to 0.443 ) more improvement in the HONOS domains emotional well-being and severity disturbance, respectively, compared with patients in non-EIP services.

Table 6 shows the results from the sensitivity analysis when changing the FTE that patients work after getting employed from 0.65 (main analysis) to 0.5 and 0.8 as well as when the eligible population for EIP is changed from 16-65 years old (main analysis) to 16-35 years old. In the most conservative scenario (target population: 16-35 year olds; FTE $=0.5$ ), the total savings/gains to overall English economy are $£ 36.6$ (95\% CI 17.7 to $55.7)$ million, of which $£ 23(95 \%$ CI 7.0 to 38.7$)$ in healthcare. In the most optimistic scenario (target population: $16-65$ year olds; $\mathrm{FTE}=0.8$ ), the total savings/gains to overall English economy are $\sim 668$ (95\% CI 31.7 to 104.5$)$ million.

\section{DISCUSSION}

EIP services are highly valued by service users and families as being non-stigmatising, recovery-focused services. $^{15} 16$ The recent Schizophrenia Commission (2012) concluded that EIP services were 'The most positive development in mental health services since the beginning of community care ... nowhere else have we seen the constant high standards, recovery ethos, co-production and multi-disciplinary team working'. Our study demonstrates that being treated within an EIP service is associated with reduced LOS in a mental

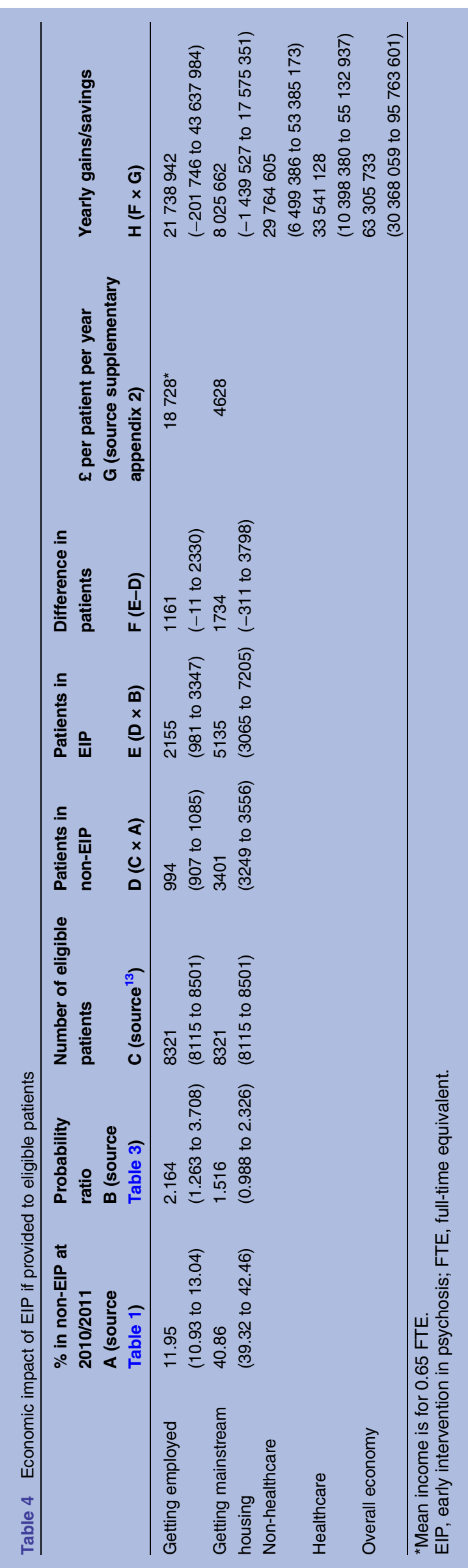


Table 5 Results from the complete case analysis and HONOS effect size

\begin{tabular}{|c|c|c|c|c|c|}
\hline Outcome & Probability ratio & SE & p Value & Lower $95 \%$ Cl & Upper $95 \% \mathrm{Cl}$ \\
\hline \multicolumn{6}{|l|}{ Complete case analysis } \\
\hline Becoming employed $(n=1801)$ & 1.989 & 0.534 & 0.011 & 1.174 & 3.368 \\
\hline Resuming studying $(n=1664)$ & 1.495 & 0.572 & 0.293 & 0.707 & 3.164 \\
\hline Moving to mainstream housing $(n=1989)$ & 1.419 & 0.262 & 0.059 & 0.987 & 2.039 \\
\hline No longer homeless $(n=1993)$ & 1.613 & 0.440 & 0.080 & 0.945 & 2.754 \\
\hline HONOS personal well-being improvement (2381) & 0.997 & 0.055 & 0.957 & 0.895 & 1.111 \\
\hline HONOS emotional well-being improvement (2381) & 1.175 & 0.053 & 0.000 & 1.075 & 1.285 \\
\hline HONOS social well-being improvement (2381) & 1.065 & 0.056 & 0.231 & 0.961 & 1.181 \\
\hline HONOS personal well-being improvement (2381) & 1.030 & 0.056 & 0.595 & 0.925 & 1.146 \\
\hline \multicolumn{6}{|c|}{ Effect size of EIP on the HONOS dimension after multiple imputation $(n=3674)$} \\
\hline HONOS personal well-being & $0.151^{*}$ & 0.153 & 0.324 & -0.149 & 0.451 \\
\hline HONOS emotional well-being & $0.545^{*}$ & 0.128 & 0.000 & 0.294 & 0.795 \\
\hline HONOS social well-being & $0.274^{*}$ & 0.158 & 0.083 & -0.036 & 0.584 \\
\hline HONOS severe disturbance & $0.246^{\star}$ & 0.101 & 0.015 & 0.048 & 0.443 \\
\hline
\end{tabular}

${ }^{*}$ These are the coefficients on a linear continuous scale.

EIP, early intervention in psychosis; HONOS, Health of the Nation Outcome Scale.

health hospital, increased contact with a CMHT and an increased chance of becoming employed, improving accommodation status and increasing emotional well-being.

EIP was estimated to save the NHS £4031 per patient per year and has the potential to generate substantial gains and savings to the overall economy. These findings were derived from a large data set, consisting of 3674 young people with psychosis who had been in contact with mental health services for 3 years between 2010 and 2013 and included 831 who had been in contact with an EIP service. These data also confirm the significant difficulties that young people with a diagnosis of psychosis experience. Only 12\% of young people with psychosis were in employment at the start of follow-up, and about $7 \%$ were in education. This is in comparison with the overall unemployment rate in the Thames Valley at this time, of between $4.5 \%$ and $6 \%$.

The cost savings for those in EIP services were seen for each of the 3 years of follow-up, with greater cost savings in years 2 and 3 than in year 1, indicating that there is continued benefit throughout the 3 years of treatment. Other studies that examined the legacy effects of EIP have found that the positive effects of 2 years of an EIP service on clinical symptoms and functioning are no longer significant at 5-year follow-up. ${ }^{17} 18$ Indeed, services that offer continuity of specialised care for 5 years albeit with a reduced intensity of intervention after the initial 2 years demonstrate sustained improvement in symptoms and functioning at 5-year follow-up relative to standard care. ${ }^{19}$ Taken together, these findings suggest that EIP is an effective intervention as long as it is continued.

Our data examined the impact of EIP services as implemented in clinical practice. This includes a level of variability within services in the fidelity to the gold standard service model of EIP treatment being delivered. This has been exacerbated in recent years with the lack of protected budgets for EIP services and the need for cost savings. Based on a preliminary analysis, there were indications about the positive relation between EIP fidelity and outcomes, but we plan to investigate this relation thoroughly in the future by using more complete and comprehensive data. However, in spite of these limitations, we can still demonstrate the large and significant impact of these services on healthcare costs and outcomes.

Our approach is in line with the Medical Research Council (MRC) guidance about using natural experiments to evaluate population health interventions. ${ }^{20}$ Following the guidance, PSM was used to introduce an experimental element in our observational study and probabilistic analysis followed by several sensitivity analyses addressing uncertainty in the results. EIP was found to increase the likelihood for improvement in emotional well-being, which supports the findings that EIP increases also the probability of moving to mainstream housing, although it was of borderline statistical significance. This is further supported by sensitivity analyses showing EIP to have a small to medium size effect (ie, between 0.20 and 0.79$)^{10}$ on emotional well-being and severity disturbance. Furthermore, adjusting for the time interval between the earliest and most recent measurements of outcomes in the analysis provided similar results.

The strengths of this current study include the large data set, the linkage of data across care settings and the statistical techniques used to analyse panel data and to control for confounding. We counted any record of contact with an EIP service during the 3 years as being treated under EIP for the whole 3-year period for the purpose of the analysis. A limitation of our analysis is that we have inferred a diagnosis of first-episode psychosis in a majority of the patients as this was not directly recorded in the electronic patient records at the time of the study. While we feel we have controlled for variables between the EIP and non-EIP groups with the data 


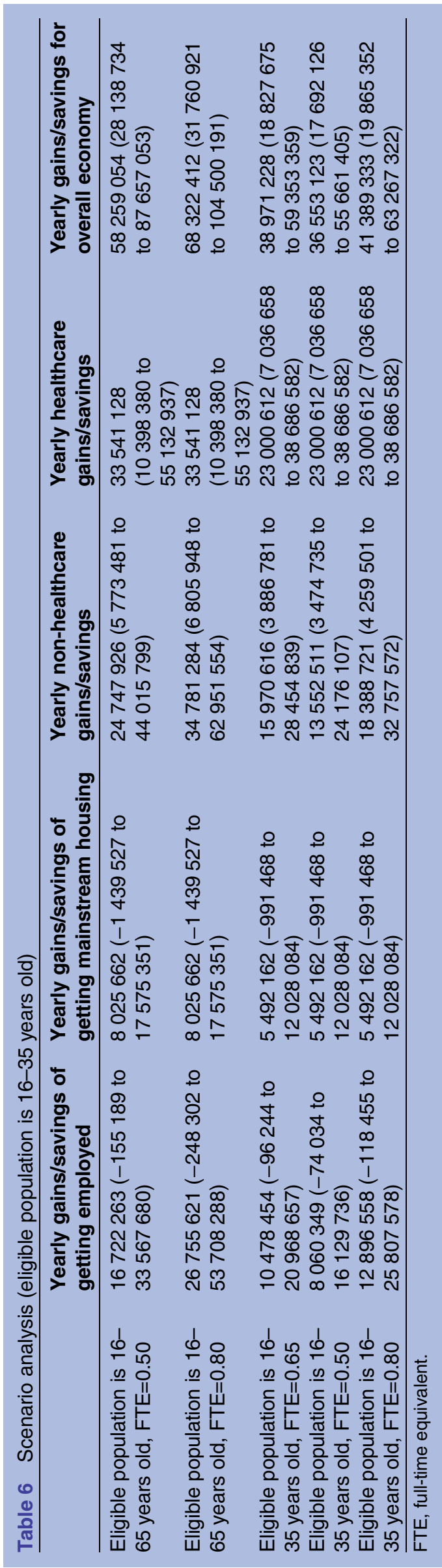

available, the direct comparability of the groups cannot be established conclusively, as would have been the case in a trial setting. Another limitation of using routine data is the lack of quality-of-life measurements that are recorded. Furthermore, in our cost calculations, we have not included the costs of setting up and running EIP services as standalone teams. Nonetheless, we are capturing the healthcare downstream costs of implementing the EIP services. Future cost-effectiveness studies of EIP services should include patient health outcome measurements and intervention costs, as well as assessment of the fidelity of services to the EIP model and detail of the interventions provided, to try and identify the necessary components for a successful outcome.

\section{CONCLUSION}

Our study suggests that EIP services, as implemented in the healthcare system in England, are associated with better health and social outcomes, and reduced costs. They could save the NHS up to $£ 33.5$ million per year and could potentially save/contribute to the English society an additional £29.8 million per year (total of $\sim £ 63.3$ million per year).

Data Raw data used for the analysis were licenced from the HSCIC by Janssen Healthcare Innovation. Initial extraction, preliminary analysis and summary data generation was performed by $\mathrm{TT}$, under the supervision of $\mathrm{BL}$ and AT. AT performed further statistical analyses using summary data. At no point were raw data accessed by anyone other than the licensee (TT). All authors take responsibility for the integrity of the data extraction and the accuracy of the data analysis.

Acknowledgements The authors would like to thank Mike Denis and Sarah Amani from Oxford AHSN, Oxford, UK for their assistance in facilitating the initial project collaboration.

Contributors BRL, AT, JL and TT designed the study. TT, JL and AT undertook data analysis, and BRL, AT, JL and TT drafted and edited the paper. BRL acts as guarantor for the study.

Funding This research was funded by the National Institute for Health Research Collaboration for Leadership in Applied Health Research and Care Oxford at Oxford Health NHS Foundation Trust and Oxford AHSN. The views expressed are those of the author(s) and not necessarily those of the NHS, the NIHR, the Department of Health or Johnson \& Johnson. The funder had no role in the collection, analysis or interpretation of data, in the writing of the report or in the decision to submit the article for publication.

Competing interests None declared.

Provenance and peer review Not commissioned; externally peer reviewed.

Data sharing statement No additional data are available.

Open Access This is an Open Access article distributed in accordance with the Creative Commons Attribution Non Commercial (CC BY-NC 4.0) license, which permits others to distribute, remix, adapt, build upon this work noncommercially, and license their derivative works on different terms, provided the original work is properly cited and the use is non-commercial. See: http:// creativecommons.org/licenses/by-nc/4.0/

\section{REFERENCES}

1. Craig TK, Garety P, Power P, et al. The Lambeth Early Onset (LEO) Team: randomised controlled trial of the effectiveness of specialised care for early psychosis. BMJ 2004;329:1067. 
2. Grawe RW, Falloon IR, Widen JH, et al. Two years of continued early treatment for recent-onset schizophrenia: a randomised controlled study. Acta Psychiatr Scand 2006;114: 328-36.

3. Health NCCfM. Psychosis and schizophrenia in adults: treatment and management. Manchester: NICE Clinical Guideline, 2014.

4. NHS England. Guidance to support the introduction of access and waiting time standards for mental health services in 2015/16. NHS England, 2015.

5. McCrone P, Craig TKJ, Power P, et al. Cost-effectiveness of an early intervention service for people with psychosis. Br J Psychiatry 2010;196:377-82.

6. Hastrup LH, Kronborg C, Bertelsen M, et al. Cost-effectiveness of early intervention in first-episode psychosis: economic evaluation of a randomised controlled trial (the OPUS study). Br J Psychiatry 2013;202:35-41.

7. Oxford Academic Health Science Network. 2016. http://www. oxfordahsn.org/

8. Network EliPI. 2016. http://www.iris-initiative.org.uk/

9. HSCIC. Quality and Outcomes Framework 2012/13. http://content. digital.nhs.uk/catalogue/PUB12262

10. Speak B. Evaluating the effectiveness of care packages with the Speal et al. 4 factor model of the HoNOS: Care Pathways and Packages Project. 2013. http://www.cppconsortium.nhs.uk/admin/ files/1373297630GUIDANCE\%20FOR\%20USE\%20OF\%204\% 20FACTOR\%20MODEL\%20OF\%20HONOS\%20AT\%20CLUSTER \%20EPISODE\%20LEVELv2.pdf

11. Stuart EA. Matching methods for causal inference: a review and a look forward. Stat Sci 2010;25:1-21.
12. Xu S, Ross $C$, Raebel MA, et al. Use of stabilized inverse propensity scores as weights to directly estimate relative risk and its confidence intervals. Value Health 2010;13:273-7.

13. Psymaptic. Psymaptic. http://www.psymaptic.org/ (accessed 1 Jan 2016).

14. Lloyd-Evans B, Marwaha S, Burns T, et al. The nature and correlates of paid and unpaid work among service users of London Community Mental Health Teams. Epidemiol Psychiatr Sci 2013;22:169-80.

15. Lester $\mathrm{H}$, Marshall $\mathrm{M}$, Jones $\mathrm{P}$, et al. Views of young people in early intervention services for first-episode psychosis in England. Psychiatr Serv 2011;62:882-7.

16. Lavis $\mathrm{A}$, Lester $\mathrm{H}$, Everard $\mathrm{L}$, et al. Layers of listening: qualitative analysis of the impact of early intervention senvices for first-episode psychosis on carers' experiences. Br J Psychiatry 2015;207:135-42.

17. Bertelsen $M$, Jeppesen $\mathrm{P}$, Petersen $\mathrm{L}$, et al. Five-year follow-up of a randomized multicenter trial of intensive early intervention vs standard treatment for patients with a first episode of psychotic illness: the OPUS trial. Arch Gen Psychiatry 2008;65:762-71.

18. Gafoor R, Nitsch D, Craig T, et al. Does early intervention (EI) in schizophrenia improve long term outcome? Results from 5 year follow-up study (South London Interventions in First Episode psychosis-LIFE). Early Interv Psychiatry 2008;2:A6.

19. Norman RM, Manchanda R, Malla AK, et al. Symptom and functional outcomes for a 5 year early intervention program for psychoses. Schizophr Res 2011;129:111-15.

20. Craig P, Cooper C, Gunnell D, et al. Using natural experiments to evaluate population health interventions: new Medical Research Council guidance. J Epidemiol Community Health 2012;66:1182-6. 